\title{
Intervención educativa sobre la enfermedad renal crónica en atención primaria
}

\author{
Cristina Boté i Fernández - Diplomada en Enfermería, Licenciada en Antropología
}

CAP Turó. SAP Muntanya (Barcelona) - Institut Català de la Salut.

\section{Sra. Directora:}

Coincidiendo con el progresivo envejecimiento de la población y la frecuente presencia de hipertensión arterial y diabetes mellitus, estamos asistiendo a una prevalencia cada vez mayor de enfermedad renal crónica (ERC) entre la población adulta, especialmente en la más añosa.

Un número importante de pacientes con ERC están sin diagnosticar. Algunos estudios subrayan que la detección y manejo precoz de la ERC y la adecuada remisión al nefrológo en los casos indicados puede disminuir la morbimortalidad de estos pacientes y reducir los costes sanitarios ${ }^{1}$.

En nuestra experiencia, no es infrecuente en el ámbito de Atención Primaria que pacientes diagnosticados de patología crónica desconozcan su enfermedad lo que podría dificultar un manejo óptimo. Por todo ello nos propusimos llevar a cabo una intervención educativa sobre la ERC en pacientes añosos de nuestro entorno y evaluar si mejoraba el conocimiento sobre la ERC tras dicha intervención.

\section{Pacientes y Métodos}

Se invitó a los ancianos usuarios de un centro social situado en el área de influencia del Centro de Asistencia Primaria (CAP) Turó (Barcelona) a asistir voluntariamente a una charla de 60 minutos sobre "Los riñones y las enfermedades renales" impartida

\begin{tabular}{|c|}
\hline $\begin{array}{c}\text { Correspondencia: } \\
\text { Cristina Boté }\end{array}$ \\
Apartado de Correos 28 \\
08480 L'Ametlla del Vallés, Barcelona \\
cbotei.bcn.ics@gencat.cat \\
\hline
\end{tabular}

por una enfermera del CAP con formación previa en Nefrología. En la acción educativa se abordaron los siguientes puntos: funciones de los riñones; cómo se diagnostica la insuficiencia renal; causas de insuficiencia renal; relación entre hipertensión, diabetes y enfermedad renal; hábitos saludables y hábitos tóxicos; medidas de prevención de la ERC y de su progresión; consecuencias de la evolución de la ERC hasta fases avanzadas y tratamiento sustitutivo renal.

Asistieron 30 personas, a las que antes de la charla informativa se les pidió que rellenasen un formulario con preguntas sobre su edad, nivel de estudios, hábito tabáquico, si padecían hipertensión o diabetes, si tomaban antihipertensivos, el tipo de dieta que realizaban, la práctica de ejercicio físico o si conocían que tuviesen insuficiencia renal crónica o alguna enfermedad renal. Además se pasó antes y después de la intervención educativa un cuestionario sobre conocimientos de la ERC con 6 preguntas de respuesta múltiple (Tabla 1), de las cuales eran válidas una 0 dos de las cinco posibles opciones de respuesta. Se puntuó cada ítem con 2 puntos si la respuesta era correcta, 1 punto si era correcta pero incompleta y 0 puntos si era incorrecta o no se contestó.

\begin{tabular}{|l|l|}
\hline $\mathbf{N}^{\mathbf{0}}$ & Pregunta \\
\hline P1 & ¿Para qué sirven los riñones? \\
\hline P2 & $\begin{array}{l}\text { ¿Qué enfermedades empeoran el funcionamiento de los } \\
\text { riñones y pueden causar una ERC? }\end{array}$ \\
\hline P3 & $\begin{array}{l}\text { ¿Qué quiere decir que una persona tiene una ERC ter- } \\
\text { minal? }\end{array}$ \\
\hline P4 & $\begin{array}{l}\text { ¿Qué puede hacer usted para evitar que empeore el fun- } \\
\text { cionamiento de sus riñones? }\end{array}$ \\
\hline P5 & $\begin{array}{l}\text { ¿Qué pasa cuando los riñones dejan de funcionar total- } \\
\text { mente porque la ERC está muy avanzada? }\end{array}$ \\
\hline P6 & ¿Cómo se puede detectar la ERC? \\
\hline
\end{tabular}

Tabla 1. Preguntas del cuestionario de conocimientos sobre enfermedad renal crónica 
Se solicitó el consentimiento informado oral para recopilar y analizar los datos de los cuestionarios y para recoger de las historias clínicas del CAP la última creatinina sérica, con la que se calculó el filtrado glomerular estimado mediante la fórmula del MDRD². Se definió insuficiencia renal crónica (IRC) como la ERC

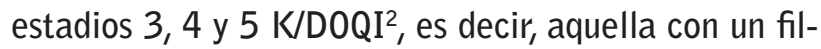
trado glomerular estimado inferior a $60 \mathrm{ml} / \mathrm{min} / 1,73$ $\mathrm{m}^{2}$. Se aseguró la confidencialidad de los datos.

La base de datos recogida fue analizada con el programa estadístico SPSS for Windows 17 . Se realizó estadística descriptiva de las características de los individuos participantes: frecuencias para las variables categóricas y medias \pm desviación estándar de las variables categóricas. Se compararon las puntuaciones de los cuestionarios antes y después de la intervención educativa usando como prueba no paramétrica el test de Wilcoxon. Se definió como nivel de significación estadística una $\mathrm{P}<0,05$.

\section{Resultados}

Participaron 30 individuos cuyas características basales están recogidas en la Tabla 2. Cabe destacar que de los 13 pacientes con IRC, sólo uno era consciente de ello ( $8 \%$ ); igualmente de los 16 pacientes que tomaban hipotensores, 3 no reconocían sufrir hipertensión arterial (19\%).

\begin{tabular}{|l|c|}
\hline $\mathbf{N}$ & 30 \\
\hline Sexo (M/F), $\mathrm{n}$ & $27 / 3$ \\
\hline Edad, años & $74 \pm 6(63-87)$ \\
\hline Nivel de estudios, \% & 21 \\
- Analfabetos & 62 \\
- Primarios & \\
\hline Creatinina sérica ${ }^{*}, \mathrm{mg} / \mathrm{dl}$ & $1,2 \pm 0,9(0,5-4,4)$ \\
Filtrado glomerular estimado por MDRD*, $^{*}$ & $52,1 \pm 14,9(12,9-60)$ \\
ml/min/1,73 m $^{2}$ & \\
\hline Tabaquismo,\% & 3 \\
- Fumadores activos & 10 \\
\hline - Exfumadores & 70 \\
\hline Ejercicio físico $\geq 1$ vez por semana, \% & 13 \\
\hline Diabéticos, \% & 55 \\
\hline Hipertensos, \% & \\
\hline Enfermedad renal crónica, \% & 56 \\
- Sin insuficiencia renal & 33 \\
- ERC estadio 3 K/D0QI & 0 \\
- ERC estadio 4 K/D0QI & 10 \\
- ERC estadio 5 K/D0QI sin diálisis & 0 \\
\hline - ERC estadio 5 K/D0QI en diálisis & \\
\hline
\end{tabular}

* media \pm desviación estándar (rango)

Tabla 2. Características de los individuos participantes en el estudio
La Tabla 3 refleja las respuestas a las diferentes preguntas del cuestionario sobre la enfermedad renal crónica, antes y después de la intervención educativa. Tras la intervención educativa un $80 \%$ de los individuos participantes (intervalo de confianza 95\%: 66-94\%) mejoraron significativamente la puntuación del cuestionario de conocimientos sobre la ERC,

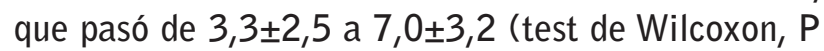
$<0,001)$.

\begin{tabular}{|c|c|c|c|c|}
\hline $\begin{array}{c}\mathbf{N}^{0} \\
\text { pregunta }\end{array}$ & $\begin{array}{c}\% \text { respuestas } \\
\text { correctas } \\
\text { pre- } \\
\text { intervención }\end{array}$ & $\begin{array}{c}\% \text { respuestas } \\
\text { correctas } \\
\text { post- } \\
\text { intervención }\end{array}$ & $\begin{array}{c}\% \text { pacientes } \\
\text { que } \\
\text { mejoraron la } \\
\text { puntuación } \\
\text { tras la } \\
\text { intervención }\end{array}$ & $\begin{array}{c}\text { P } \\
\text { (test de } \\
\text { Wilcoxon) }\end{array}$ \\
\hline P1 & 3 & 17 & 23 & 0,475 \\
\hline P2 & 3 & 73 & 77 & 0,001 \\
\hline P3 & 30 & 66 & 43 & 0,005 \\
\hline P4 & 6 & 17 & 37 & 0,03 \\
\hline P5 & 37 & 70 & 50 & 0,068 \\
\hline P6 & 6 & 57 & 50 & 0,001 \\
\hline \multicolumn{3}{|c|}{ TOTAL } & 80 & 0,001 \\
\hline
\end{tabular}

Tabla 3. Respuestas correctas al cuestionario de conocimientos sobre la enfermedad renal crónica antes y después de la intervención educativa

\section{Discusión}

La ERC es una gran desconocida, incluso entre los pacientes que la padecen, que a menudo no son conscientes de ello. Una intervención educativa sencilla como la aquí utilizada puede mejorar el conocimiento de la ERC entre la población general añosa, más susceptible de padecerla. No obstante, las experiencias previamente publicadas en nuestro medio sobre educación sanitaria en pacientes con IRC suelen darse en el ámbito de los servicios de Nefrología hospitalarios o en los centros de diálisis, más que en los centros de Atención Primaria ${ }^{3-5}$.

Para poder facilitar la adopción de medidas de prevención de la progresión de la insuficiencia renal en estos pacientes así como mejorar la adherencia terapéutica ${ }^{6}$, primero es preciso que los pacientes conozcan que padecen una ERC, por lo que creemos que la información y la educación sanitaria podrían desempeñar un papel importante ya desde la Atención Primaria ${ }^{7}$. 


\section{Bibliografía}

1. Aguilar MD, Orte L, Lázaro P, Gómez-Campderá F, Fernández E, Sanz D. En representación del Grupo INESIR i Pastor V. Eficiencia de implantar en atención primaria un programa dirigido a conseguir la referencia precoz al nefrólogo de los pacientes con insuficiencia renal crónica. Nefrología 2006; 26(Supl 3):114-120.

2. National Kidney Foundation. K/DOQI Clinical practice guidelines for chronic kidney disease: evaluation, classification and stratification. Am J Kidney Dis 2002; 39(2 Suppl 1):S1-266.

3. Muriana C, Puigvert C, García ML. Valoración de un programa de educación sanitaria a pacientes con insuficiencia renal crónica. Rev Soc Esp Enferm Nefrol 2002; 5(4):14-17.

4. Casado MA, Martínez M, Jara J, Martínez A. Educación sanitaria en la insuficiencia renal crónica. En: Libro de comunicaciones presentadas al XXVIII Congreso Nacional de la Sociedad Española de Enfermería Nefrológica; Palma de Mallorca, 15-18 octubre 2003. Disponible en: http://www. revistaseden.org/files/art64_1.doc

5. Martínez S, Gago MC, Gruss E, Garrido MV, Andrés MM, Rubio $E$, Royuela $C$, Marco $B$, Moreno JA. Atención primaria en el paciente renal: importancia de enfermería nefrológica en la prevención, promoción y educación para la salud. En: Libro de comunicaciones del XXX Congreso Nacional de la Sociedad Española de Enfermería Nefrológica; Málaga, 12-15 octubre 2005. Disponible en: http://www.revistaseden.org/files/ atencion\%20primaria.pdf

6. Ronda MJ. Prevención de la insuficiencia renal. Atención primaria de enfermería. En: Andrés J, Fortuny C. Cuidados de Enfermería en la Insuficiencia Renal. Madrid: ELA; 1994: 43-51.

7. Dasí MJ, Rojo E. Continuidad de los cuidados de enfermería: necesidad de coordinación entre niveles. Rev Soc Esp Enferm Nefrol 2000; 3(2):27-33. 